\title{
Three Perspectives of Granular Computing
}

\author{
Yiyu (Y.Y.) Yao \\ Department of Computer Science, University of Regina \\ Regina, Saskatchewan, Cananda S4S 0A2 \\ E-mail: yyao@cs.uregina.ca \\ URL: http://www.cs.uregina.ca/ yyao
}

\begin{abstract}
As an emerging field of study, granular computing has received much attention. Many models, frameworks, methods and techniques have been proposed and studied. It is perhaps the time to seek for a general and unified view so that fundamental issues can be examined and clarified. This paper examines granular computing from three perspectives. By viewing granular computing as a way of structured thinking, we focus on its philosophical foundations in modeling human perception of the reality. By viewing granular computing as a method of structured problem solving, we examine its theoretical and methodological foundations in solving a wide range of realworld problems. By viewing granular computing as a paradigm of information processing, we turn our attention to its more concrete techniques. The three perspectives together offer a holistic view of granular computing.
\end{abstract}

Keywords: granular computing, structured thinking, structured problem solving, information processing paradigm

\section{INTRODUCTION}

The term "granular computing (GrC)" is used by researchers in computational intelligence as a name to label an emerging field of interdisciplinary study that explores different levels of granularity in human-centered problem solving and information processing [2,3,7$9,11,12,17-29,31,32]$. In the past few years, many theories, frameworks, models, methodologies, and techniques have been proposed and investigated. Due to its widespread uses and a large diversity of interpretations and formulations, many authors believe that it is necessary to answer the fundamental questions that differentiate granular computing as a separate field in its own right $[2,24,25]$.

The fundamental ideas and principles of granular computing have long appeared in many branches of sciences [2,3,8,12,23-25,31,32]. It is therefore not surprising to find that some studies are simply a restatement of existing results by using the terminologies of granular computing. Many studies suffer from unnecessary domain dependent details. In order to fully appreciate its universal applicability, flexibility, and effectiveness, one can study granular computing at multiple levels of detail, by applying its own principles [24,25].

The following two quotes serve as the basis of the present investigation on the nature and the scope of granular computing.

"Granular computing, in our view, attempts to extract the commonalities from existing fields to establish a set of generally applicable principles, to synthesize their results into an integrated whole, and to connect fragmentary studies in a unified framework. Granular computing at philosophical level concerns structured thinking, and at the application level concerns structured problem solving. While structured thinking provides guidelines and leads naturally to structured problem solving, structured problem solving implements the philosophy of structured thinking." (Yao, 2005, Perspectives of Granular Computing [25])

"We propose that Granular Computing is defined as a structured combination of algorithmic abstraction of data and non-algorithmic, empirical verification of the semantics of these abstractions. This definition is general in that it does not specify the mechanism of algorithmic abstraction nor it elaborates on the techniques of experimental verification. Instead, it highlights the essence of combining computational and non-computational information processing." (Bargiela and Pedrycz, 2006, The roots of Granular Computing [2])

Granular computing results in a powerful point of view of human understanding and problem solving, which is perhaps more important than any of its specific and concrete methods. It is necessary to have a holistic and unified view of granular computing, in contrast to fragmentary and disjointed studies. In what follows, we argue that such a whole view may be achievable by looking at the three levels or the three perspectives:

- At the philosophical level, granular computing is viewed as a way of structured thinking.

- At the practical level, granular computing is viewed as a general method of structured problem solving.

- At the computational level, granular computing is viewed as a paradigm of information processing.

By separating and focusing on different types of issues at the three levels, we may be a step closer to an in-depth and a multi-aspect understanding of granular computing.

\section{GRANUlAR COMPUTING AS A WAY OF STRUCTURED THINKING}

Hierarchical organizations and structures are abundant in the real world $[1,3,15,23,31]$. They can be found in many natural, artificial and man-made systems. Human perception and understanding of the real world depends, to a large extent, on such nested and hierarchical structures $[1,15,23]$. A key notion for studying hierarchical structures is the granularity at multiple levels that are defined through the abstraction, control, complexity, detail, resolution, and so on. This leads to a philosophical foundation of granular computing. One needs to establish general principles and philosophy in order to more appropriately perceive, describe, understand, and describe the reality. From the philosophical perspective, granular computing is in fact a 
Yao, Y.Y. Three perspectives of granular computing

The Proceedings, International Forum on Theory of GrC from Rough Set Perspective Journal of Nanchang Institute of Technology, Vol. 25, No. 2, pp. 16-21, 2006.

way of structured thinking. It offers general domain independent principles and views for understanding the real world and problems in the real world using different levels of granularity.

Human understanding of the physical world is conceptually summarized as knowledge. The hierarchical structures that exist in the real world are naturally reflected in the hierarchical organization of human knowledge $[14,16,23]$. Natural and formal languages that we use to represent, describe and communicate human knowledge have well-equipped tools for dealing with granularity. For example, specific words typically represent smaller granules and general words represent larger granules. Typical scientific writings that document and communicate human knowledge can be easily investigated based on different levels of granularity. The title of an article is a brief description at a high level of granularity. Lower levels of granularity are given by section titles, subsection titles, and so on. The most detailed level is the entire text of the article. The abstract is another description of the article at a high level of granularity.

A few exemplary studies related to knowledge granularity and human processing of such granular knowledge are given below. They provide evidence supporting granular computing as structured thinking at the philosophical level.

In teaching problem solving in physics, Reif and Heller [14] state that "effective problem solving in a realistic domain depends crucially on the content and structure of the knowledge about the particular domain". Knowledge about physics in fact specifies concepts and relations between them at various levels of abstraction. Furthermore, the knowledge is organized hierarchically, with explicit guidelines specifying when and how this knowledge is to be applied. Posner [13] suggests that, according to the cognitive science approach, to learn a new field is to build appropriate cognitive structures and to learn to perform computations that will transform what is known into what is not yet known.

In the book "On Intelligence", Hawkins [4] uses the notion of a cortical hierarchy for deriving a memory-prediction framework for explaining intelligence. In this top-down approach for understanding the brain, a conceptual model of cortex is given by highlighting its hierarchical connectivity and information flows up and down the hierarchy. The model is based on the observations: “... the cortex's hierarchical structure stores a model of the hierarchical structure of the real world. The real world's nested structure is mirrored by the nested structure of our cortex."

Wang et al. [30] propose a layered reference conceptual model of the brain, in which the brain is modeled as a multi-level hierarchy based on various brain functions, abilities and activities. It is argued that different level brain functions deal with different tasks. One may also link those tasks to different levels of granularity.

In summary, granular computing, as structured thinking, explores multi-level granularity that exists in the physical world. This philosophy is consistent to, and reflects nicely, the reality. Granular computing helps us to arrive at accurate and natural description, as well as in-depth understanding, of the inherent structures and complexity of the real world.

\section{GRANUlar COMPUTING AS A Method OF StRUCTURED PROBLEM SOLVING}

From the practical perspective, granular computing concerns structured problem solving [5,33,34]. It promotes a general approach for describing, understanding, analyzing, examining, and solving real world problems based on a multiple level organization through abstraction, control, complexity, detail, resolution, and so on. In this

perspective, granular computing can be better explained with references to results in artificial intelligence $[5,33,34]$.

Hobbs [5] proposes a theory of granularity that indeed captures some of the essential features of granular computing as a method of structured problem solving. We perceive and represent the world under various grain sizes, and abstract only those things that serve our present interests. The ability to conceptualize the world at different granularities and to switch among these granularities is fundamental to our intelligence and flexibility. This enables us to map the complexities of real world into computationally tractable simpler theories.

State space representation and search is a well-known method for problem solving developed in artificial intelligence. By applying the notions of hierarchical structures and granularity to state spaces, Zhang and Zhang $[33,34]$ introduce a quotient space theory. It is a natural generalization of the standard state-space method. One derives a granulated state space as a multi-level description and representation of a problem, which allows efficiently search to obtain a solution to the problem. The quotient space theory serves as a good example that motivates us to view granular computing as a general method of structured problem solving.

The philosophical perspective of granular computing suggests the need for structured thinking with hierarchical modeling. The generation and utilization of hierarchical organizations and structures at a more practical level rely on a strategy known as the divide and conquer. With this strategy, a problem described with larger granules is decomposed into a family of sub-problems described with smaller granules, and the solution of the problem is obtained by combining the solutions of sub-problems. Structured programming is one of the best examples to illustrate such a strategy.

The principles and characteristics of the top-down design and stepwise refinement of structured programming, taken from Ledgard, Gueras and Nagin [6], are given as:

- design in levels;

- $\quad$ initial language independence;

- postponement of details to lower levels;

- formalization of each level;

- verification of each level; and

- $\quad$ successive refinements.

It is an easy task to apply the same principles elsewhere. For example, it has been suggested that the top-down approach is effective for developing, communicating and writing mathematical proofs. The same principles can be applied in the preparation, organization, and writing of scientific articles. More examples can be found in [23].

The practical wisdom of granular computing leads to a general method of structured problem solving. Unfortunately, those principles and ideas have been widely applied across many disciplines under various different names, and in many cases are buried in much detailed domain dependent techniques. They are not readily available in a more generic form. The extraction of a set of more general and domain independent principles under the umbrella of granular computing may prove to be even more useful. The abstract high-level ideas and points of view may enable us to build eventually a methodological foundation for scientific discovery and enquiry in many branches of science. 
Yao, Y.Y. Three perspectives of granular computing

The Proceedings, International Forum on Theory of GrC from Rough Set Perspective

Journal of Nanchang Institute of Technology, Vol. 25, No. 2, pp. 16-21, 2006.

\section{GRANUlAR COMPUTING AS A PARADIGM OF INFORMATION PROCESSING}

From the computational perspective, granular computing focuses on a paradigm for representing and processing information in a multiple level architecture [2,3]. As suggested by Bargiela and Pedrycz [2], granular computing can be viewed as a structured combination of algorithmic and non-algorithmic information processing. The information-processing paradigm will lead to more effective and concrete computational models.

The two basic ingredients of the information-processing paradigm are representation and process [2,3]. Both of them can be studied according to the semantics (i.e., non-algorithmic) and the algorithmic aspects [2,3,20-25]. A computational model of granular computing involves many issues surrounding the two notions. Based on discussions in reference [26], some of the issues are summarized:

\section{- Representation: granules}

A granule may be interpreted as one of the numerous small particles forming a larger unit. They have at least three basic properties:

- internal properties;

- external properties; and

- contextual properties.

A granule is treated both as a family of individual elements characterized by its internal properties and as an inseparable whole characterized by its external properties. The existence of a granule is only meaningful in a certain context. Elements in a granule can be themselves granules, and a granule can also be an element of another granule.

\section{- Representation: granular structures}

Granular structures provide descriptions of a system or a problem under consideration. We can identify at least three levels of structure on a web of granules:

- internal structure of a granule;

- collective structure of a family of granules; and

- hierarchical structure of a web of granules.

A collective structure of family of granules may be interpreted as a level or a granulated view in an overall hierarchical structure. Itself may be an inter-connected network of granules. For the same system or the same problem, many interpretations and descriptions may co-exist. Granular structures need to be modeled as multiple hierarchies and multiple levels in each hierarchy [25].

\section{- Process: granulation}

Granulation involves the construction of the building blocks and structures for granular computing, namely, granules, granulated views, web of granules, and hierarchies. Some of the issues are [25]:

- granulation criteria;

- granulation algorithms/methods;

- representation of granules and granular structures; and

- characterization of granule and granular structures.

\section{- Process: computing with granules}

Granular computing suggests that one can solve a problem by systematically exploring the granular structures. This involves the two-way communications up and down in a hierarchy and moving within a hierarchy. Some of the issues are:

- mappings connecting granules and levels;

- granularity conversion;
- $\quad$ operators of computing; and

- $\quad$ property preservation or invariant properties.

In the above discussion, we in fact used a two-level description: one deals with issues at the granule-level and the other deals with issues at the hierarchy-level. In general, one may further study many issues at more than two levels.

The information-processing paradigm, based on an informationprocessing pyramid with different-sized information granules, captures essential features of granular computing. When the abstract notions, such as granules, granular structures, levels, hierarchies and so on, are associated with specific physical interpretations in a particular domain, one can immediately apply granular computing approaches.

As a more concrete example for illustration, we take a look at the work of Marr [10] on vision. In an attempt to establish a computational model for human representation and processing of visual information, Marr makes a convincing argument that calls for a multi-level conceptual framework. It is demonstrated that a full understanding of an information-processing system depends crucially on different kinds of explanations at different levels of descriptions that are linked into a cohesive whole. The three levels considered are the computational theory, the representation and algorithm, and the hardware implementation levels. Furthermore, for an informationprocessing problem, it is necessary to study the twin strands of representation and process. The deep insights of Marr are extremely valuable for us to view granular computing as a paradigm of information processing.

According to Marr [10], a representation is a formal system that makes explicit certain entities or types of information and a specification on how the system does it. The result of using a representation to describe an entity is called a description of the entity in the representation. In addition, when making explicit certain information, a particular representation may have to push other information into background. In the context of granular computing, we can explore the implications of those observations. At a particular level of granularity, we can adopt a particular representation that makes explicit certain aspects of the problem and at the same time hides other aspects. The choice of a representation depends on our intention at that particular level.

A process may be simply interpreted as actions, procedures, or the like for carrying out information-processing tasks. A process manipulates certain entities based on their descriptions in a particular representation. A process normally transforms its input to output. In granular computing, granules and their structures are entities that are manipulated by processes. Processes deal with the computational information-processing tasks. The choice of an algorithm that carries out a process depends crucially on a particular representation, as well as the characteristics of the algorithm.

Based on Marr's work on vision, the study of granular computing as an information-processing paradigm must carefully examine the related notions of representation and process at different levels of granularity. As there exist many possible representations and many possible algorithms for carrying out a process, one may need to consider multiple hierarchies and multiple levels in each hierarchy in granular computing [25].

\section{CONCLUSION}

The necessity, the needs and the benefits of granular computing have been amply demonstrated by many authors. Concrete models and methodologies are constantly being proposed. There seems to be 
Yao, Y.Y. Three perspectives of granular computing

The Proceedings, International Forum on Theory of GrC from Rough Set Perspective

Journal of Nanchang Institute of Technology, Vol. 25, No. 2, pp. 16-21, 2006.

a lack of philosophical and conceptual examinations. This makes it a bit difficult to see the uniqueness of granular computing as a field of study in its own right, in spite of its very rapid development.

In several of our recent publications [22-26], we attempt to bring insights from many disciplines. This leads us to study granular computing at multiple levels with different focuses. Three perspectives are examined in this paper: granular computing as structured thinking, as structured problem solving, and as an information-processing paradigm. While structured thinking provides guidelines and leads naturally to structured problem solving, structured problem solving implements the philosophy of structured thinking. The information-processing paradigm focuses specifically on a family of concrete methods of granular computing.

To support our argument, we have drawn results from many studies. For structured thinking, we mention the notion of knowledge granularity used by Reif and Heller [14], the memory-prediction framework of Harkins [4], and the layered reference model of the brain proposed by Wang et al. [30]. It appears that structured thinking based on multiple levels of granularity matches naturally with the real world, and furthermore our brain may actually employ such a hierarchical structure. For structured problem solving, we examine the theory of granularity proposed by Hobbs [5], the quotient space theory developed by Zhang and Zhang [33,34]. We show that the general principles of structured programming [6] capture the essential features of granular computing. Bargiela and Pedrycz [2,3] promote granular computing as a new paradigm of information processing. We provide further evidence to support such a view by recalling the work of Marr [10] on the human representation and processing of visual information. We emphasize that the twin strands of representation and process need to be further investigated.

Although the three perspectives may not be exhaustive for granular computing, a careful study of them may bring us a step closer to a full understanding of granular computing. The paper is not intended to answer fully some of the fundamental questions. Instead, we hope to encourage more discussions on a whole view of granular computing.

\section{Acknowledgement}

The author would like to express his thanks to Professor Qing Liu for organizing the International Forum and inviting him for a keynote speech.

\section{REFERENCES}

[1] Ahl, V. and Allen, T.F.H. Hierarchy Theory, a Vision, Vocabulary and Epistemology, Columbia University Press, 1996.

[2] Bargiela, A. and Pedrycz, W. "The roots of granular computing," Proccedings of the 2006 IEEE International Conference on Granular Computing (GrC'06), 2006, pp. 806-809.

[3] Bargiela, A. and Pedrycz W. Granular Computing: an Introduction, Kluwer Academic Publishers, Boston, 2002.

[4] Hawkins, J. (with Blakeslee, S.) On Intelligence, Henry Holt and Company, New York, 2004.

[5] Hobbs, J.R. "Granularity," Proceedings of the Ninth International Joint Conference on Artificial Intelligence, pp. 432-435, 1985.

[6] Ledgard, H.F., Gueras, J.F. and Nagin, P.A. PASCAL with Style: Programming Proverbs, Hayden Book Company, Inc., Rechelle Park, New Jersey, 1979.

[7] Lin, T.Y. Granular computing, Announcement of the BISC Special Interest Group on Granular Computing, 1997.

[8] Lin, T.Y., Yao, Y.Y. and Zadeh, L.A. (Eds.) Data Mining, Rough Sets and Granular Computing, Physica-Verlag, Heidelberg, 2002.

[9] Liu, Q. and Wang, Q.Y. "Granular logic with closeness reltion $\sim \lambda$ and its reasoning," Proceedings of RSFDGrC'05, LNAI 3641, pp. 709-717, 2005.

[10] Marr, D. Vision: A Computational Investigation into Human Representation and Processing of Visual Information, W.H. Freeman and Company, San Francisco, 1982.

[11] Nguyen, S.H., Skowron, A., Stepaniuk, J. "Granular computing: a rough set approach," Computational Intelligence, vol. 17, pp. 514-544, 2001.

[12] Pedrycz, W. (Ed.) Granular Computing: An Emerging Paradigm, Physica-Verlag, Heidelberg, 2001.

[13] Posner, M.I. (Ed.) Foundations of Cognitive Science, The MIT Press, Cambridge, Massachusetts, 1989.

[14] Reif, F. and Heller, J. "Knowledge structure and problem solving in physics," Educational Psychologist, vol. 17, pp. 102-127, 1982.

[15] Simon, H.A. "The organization of complex systems," in: Hierarchy Theory, The Challenge of Complex Systems, Pattee, H.H. (Ed.), George Braziller, New York, pp. 1-27, 1963.

[16] Sowa, J.F. Conceptual Structures, Information Processing in Mind and Machine, Addison-Wesley, Reading, Massachusetts, 1984.

[17] Yao, J.T. and Yao, Y.Y. "Induction of classification rules by granular computing," Proceedings of the 3rd International Conference on Rough Sets and Current Trends in Computing, LNAI 2475, pp. 331-338, 2002.

[18] Yao, J.T. and Yao, Y.Y. "A granular computing approach to machine learning," Proceedings of the 1st International Conference o Fuzzy Systems and Knowledge Discovery (FSKD'02), Singapore, pp. 732-736, 2002.

[19] Yao, Y.Y. "Granular computing using neighborhood systems," in: Roy, R., Furuhashi, T., and Chawdhry, P.K. (Eds.), Advances in Soft Computing: Engineering Design and Manufacturing, Springer-Verlag, London, pp. 539-553, 1999.

[20] Yao, Y.Y. "Granular computing: basic issues and possible solutions," Proceedings of the 5th Joint Conference on Information Sciences, pp. 186-189, 2000.

[21] Yao, Y.Y. "Information granulation and rough set approximation," International Journal of Intelligent Systems, vol. 16, pp. 87-104, 2001.

[22] Yao, Y.Y. "Modeling data mining with granular computing," Proceedings of COMPSAC 2001, pp. 638-643, 2001.

[23] Yao, Y.Y. "A partition model of granular computing," LNCS Transactions on Rough Sets, vol. 1, 232-253, 2004.

[24] Yao, Y.Y. "Granular computing," Computer Science (Ji Suan Ji Ke Xue), vol. 31, pp. 1-5, 2004.

[25] Yao, Y.Y. "Perspectives of granular computing," Proceedings of 2005 IEEE International Conference on Granular Computing, vol. 1, pp. 8590, 2005.

[26] Yao, Y.Y. "Granular computing for data mining," Proceedings of SPIE Conference on Data Mining, Intrusion Detection, Information Assurance, and Data Networks Security, paper \#: 624105, 2006.

[27] Yao, Y.Y., Liau, C.-J. and Zhong, N. "Granular computing based on rough sets, quotient space theory, and belief functions," Proceedings of ISMIS'03, pp. 152-159, 2003.

[28] Yao, Y.Y. and Zhong, N. "Potential applications of granular computing in knowledge discovery and data mining," Proceedings of World Multiconference on Systemics, Cybernetics and Informatics, vol. 5, Computer Science and Engineering, pp. 573-580, 1999.

[29] Yao, Y.Y. and Zhong, N. "Granular computing using information tables," in: Lin, T.Y., Yao, Y.Y. and Zadeh, L.A. (Eds.), Data Mining, Rough Sets and Granular Computing, Physica-Verlag, Heidelberg, pp. 102-124, 2002.

[30] Wang, Y., Wang, Y., Patel, S., and Patel, D. A layered reference model of the brain (LRMB), IEEE Transactions on Systems, Man, and Cybernetics (C), vol. 36, pp. 124-133, 2006.

[31] Zadeh, L.A. "Towards a theory of fuzzy information granulation and its centrality in human reasoning and fuzzy logic," Fuzzy Sets and Systems, vol. 19, pp. 111-127, 1997.

[32] Zadeh, L.A. "Some reflections on soft computing, granular computing and their roles in the conception, design and utilization of 
Yao, Y.Y. Three perspectives of granular computing

The Proceedings, International Forum on Theory of GrC from Rough Set Perspective

Journal of Nanchang Institute of Technology, Vol. 25, No. 2, pp. 16-21, 2006.

information/intelligent systems," Soft Computing, vol. 2, pp. 23-25, 1998.

[34] Zhang, L. and Zhang, B. "The quotient space theory of problem solving," Fundamenta Informatcae, vol. 59, pp. 287-298, 2004.

[33] Zhang, B. and Zhang, L. Theory and Applications of Problem Solving, North-Holland, Amsterdam, 1992. 